\title{
Comportamento de linhagens de sorgo forrageiro submetidas a déficit hídrico sob condição controlada ${ }^{(1)}$
}

Sérgio Ribeiro do Amaral(2), Mário de Andrade Lira( ${ }^{(3)}$, José Nildo Tabosa ${ }^{(4)}$, Mércia Virginia Ferreira dos Santos ${ }^{(5)}$, Alexandre Carneiro Leão de Mello(6) e Venésio Felipe dos Santos ${ }^{(4)}$

\begin{abstract}
Resumo - O objetivo deste trabalho foi avaliar os efeitos do estresse hídrico no estabelecimento, desenvolvimento e na taxa de sobrevivência de 11 linhagens de sorgo forrageiro em casa de vegetação. O delineamento experimental foi o de blocos ao acaso no arranjo de parcelas subdivididas no tempo, com cinco repetições. As unidades experimentais foram constituídas por recipientes de $250 \mathrm{~L}$, onde foram colocados $187,9 \mathrm{~kg}$ de solo da classe textural areia franca. O estudo compreendeu dois períodos de estresse, e três irrigações, sendo uma no início e as outras aplicadas quando grande número de folhas tinham aspecto de palha seca. No primeiro período, as linhagens apresentaram alta taxa de sobrevivência, demonstrando resistência ao estresse hídrico. As linhagens que apresentaram menor velocidade de crescimento nesse período sofreram menos com o estresse. No segundo período não ocorreram diferenças significativas entre as linhagens com relação ao desenvolvimento das plantas. Durante os dois períodos de estresse, a velocidade de crescimento não diferenciou satisfatoriamente linhagens de comportamento distintos, quanto à resistência à seca, competindo em um mesmo recipiente e em alta densidade populacional. As linhagens 84, 29, 99, 41, 63 e 104 apresentaram maior resistência ao estresse hídrico e as linhagens 33, 38, 40, 67 e 92, menor resistência.
\end{abstract}

Termos para indexação: Sorghum bicolor, estresse hídrico, resistência à seca, desempenho vegetal.

\section{Forage sorghum lines submitted to water deficit stress under controlled conditions}

\begin{abstract}
The objective of this work was to evaluate the effects of water deficit stress on establishment, development and survival rate of 11 forage sorghum lines in a greenhouse. The experiment was carried out under a randomized block design, with split-splot arrangement and five replications. Each replication was formed by a container of $250 \mathrm{~L}$ capacity, filled with $187.9 \mathrm{~kg}$ of sandy soil. Two stress periods were studied, with three irrigations, at the begining of tests and when the plants showed large numbers of dry leaves. During the first period all lines had high survival rates showing resistance to water deficit stress. Slower growing lines suffered less with the imposed water deficit. During the second water deficit stress period there were no significant differences between the lines in plant development. Along both stress periods, growth rate did not distinguish satisfactorily lines with distinct behavior, as relate to drought resistance, when those lines were competing on the same recipient and high population density. Lines 84, 29, 99, 41, 63, and 104 had higher stress resistant while lines 33, 38, 40, 67, and 92 had lower stress resistant.
\end{abstract}

Index terms: Sorghum bicolor, water stress, drought resistance, plant performance.

(1) Aceito para publicação em 22 de maio de 2003. Extraído da dissertação de mestrado apresentada pelo primeiro autor à Universidade Federal Rural de Pernambuco (UFRPE), Recife, PE.

(2) UFRPE, Av. Dom Manoel de Medeiros, s/noำ, Dois Irmãos, CEP 52171-030 Recife, PE.

E-mail: srdoamaral@aol.com.br
(3) Empresa Pernambucana de Pesquisa Agropecuária (IPA), Av. General San Martin, 1371, Bongi, CEP 50761-000 Recife, PE. Bolsista do CNPq. E-mail: mlira@ipa.br

(4) IPA. E-mail: tabosa@ipa.br, venesio@ipa.br

(5) UFRPE, Dep. de Zootecnia. Bolsista do CNPq.

E-mail: rmsantos@elogica.com.br

(6) UFRPE, Dep. de Zootecnia. Bolsista da Facepe.

E-mail: melloacl@ig.com.br 


\section{Introdução}

O Nordeste brasileiro compreende uma área de $1.548 .672 \mathrm{~km}^{2}$, da qual 52\% corresponde à região do Semi-Árido, com secas periódicas que afetam sua estrutura social e econômica (Lira et al., 1989). Resposta das culturas à variação de níveis hídricos tem sido propósito de pesquisas científicas, buscando o aumento na eficiência do uso de água pelas plantas, com vistas à otimização de práticas de manejo, bem como ao maior entendimento dos efeitos do estresse hídrico no crescimento e na produção de matéria seca (Gomide et al., 1998).

A cultura do sorgo (Sorghum bicolor (L.) Moench) apresenta grande potencial de produção de forragem na Região Nordeste, por sua capacidade de adaptação, tolerância a temperaturas elevadas e também por possuir características de xerofilia (Reis, 1992). Esse diferencial é importante em sistemas de produção em regiões sujeitas a secas e que não disponham de irrigação (Oliveira et al., 2002).

O sorgo possui características fisiológicas que permitem paralisar o crescimento ou diminuir as atividades metabólicas durante o estresse hídrico e reiniciar o crescimento quando a água se torna disponível (Masojidek et al., 1991). Além de que, logo após o término de um período de estresse hídrico, as plantas podem até crescer mais rapidamente do que as que não sofreram estresse. Essa situação ocorre, provavelmente, pelo acúmulo de fotoassimilados no início do período de estresse. Essas reservas, que são pou- co utilizadas durante a seca, ficam disponíveis para estimular o crescimento quando a água se torna novamente disponível (Donatelli et al., 1992).

A tolerância à seca é diferenciada conforme o genótipo (Masojidek et al., 1991; Donatelli et al., 1992), dentro da mesma espécie vegetal. Assim, diferentes materiais de uma mesma espécie podem responder de maneiras distintas ao estresse causado pela deficiência hídrica. Um dos sintomas dessa deficiência mais característico apresentado pelas plantas é a murcha, que, dependendo da severidade do estresse, pode ser reversível ou não.

Este trabalho teve como objetivo avaliar os efeitos do estresse hídrico no estabelecimento, desenvolvimento e na taxa de sobrevivência de 11 linhagens de sorgo forrageiro.

\section{Material e Métodos}

O trabalho foi desenvolvido em casa de vegetação do Departamento de Zootecnia da Universidade Federal Rural de Pernambuco (UFRPE), Recife, PE, de 14/8/95 a 19/12/95. Foram avaliadas 11 linhagens de sorgo forrageiro (Tabela 1), provenientes do Programa de Melhoramento da Empresa Pernambucana de Pesquisa Agropecuária (IPA). Essas linhagens foram geradas pelo programa objetivando resistência à seca na fase de plântulas, em condições de campo, e elevado potencial de produção de forragem.

Foram utilizados cinco recipientes cilíndricos de cimento amianto, cada um com capacidade de $250 \mathrm{~L}, 0,5 \mathrm{~m}$ de altura e superfície de $0,4 \mathrm{~m}^{2}$. Em cada recipiente foram colocados $187,9 \mathrm{~kg}$ de solo, coletado no campus da UFRPE

Tabela 1. Genealogia de linhagens de sorgo forrageiro cultivadas em casa de vegetação.

\begin{tabular}{|c|c|}
\hline Linhagem & Genealogia $^{(1)}$ \\
\hline 29 & (116 x 322-1-3)-st87-32- st88-01- st89-01-st90-01-st91-01-vit91-Ca92-B-Ca94-B \\
\hline 33 & (116 x 467-1-3)-st87-02- st88-01- st89-01-st90-01-st91-01-vit91-Ca92-B-Ca94-B \\
\hline 38 & (1158 x 322-1-3)-st87-10-st88-02- st89-01-st90-01-st91-03-vit91-Ca92-B-Ca94-B \\
\hline 40 & (1158 x 322-1-3)-st87-10-st88-02- st89-01-st90-01-st91-05-vit91-Ca92-B-Ca94-B \\
\hline 41 & (1158 x 322-1-3)-st87-10-st88-02- st89-01-st90-01-st91-06-vit91-Ca92-B-Ca94-B \\
\hline 63 & (1158 x 322-1-3)-st87-11-st88-03- st89-02-st90-01-st91-09-vit91-Ca92-B-Ca94-B \\
\hline 67 & (1158 x 322-1-3)-st87-09-st88-03- st89-01-st90-01-st91-01-vit91-Ca92-B-Ca94-B \\
\hline 84 & (1158 x 322-1-3)-st87-13-st88-01- st89-01-st90-01-st91-01-vit91-Ca92-B-Ca94-B \\
\hline 92 & (1198 x 322-1-3)-st87-18-st88-01- st89-01-st90-01-st91-05-vit91-Ca92-B-Ca94-B \\
\hline 99 & (1158 x 322-1-3)-st87-18-st88-01- st89-01-st90-01-st91-13-vit91-Ca92-B-Ca94-B \\
\hline 104 & (1158 x 322-1-3)-st87-18-st88-04- st89-02-st90-01-st91-02-vit91-Ca92-B-Ca94-B \\
\hline
\end{tabular}

${ }^{(1)}$ st: Estação Experimental de Serra Talhada, PE; vit: Estação Experimental de Vitória de Santo Antão, PE; Ca: Estação Experimental de Caruaru, PE; B: coleta em bulk. 
e analisado nos laboratórios do IPA, da classe textural areia franca, com pH 7,0 e com os seguintes valores de composição: $\mathrm{P}, 36,0 \mathrm{mg} / \mathrm{dm}^{3} ; \mathrm{K}, 150,0 \mathrm{mg} / \mathrm{dm}^{3} ; \mathrm{Al}^{3+}, 0,0 \mathrm{cmol}_{\alpha} / \mathrm{dm}^{3}$; $\mathrm{Ca}^{2+}, 8,3 \mathrm{cmol}_{\mathrm{c}} / \mathrm{dm}^{3} ; \mathrm{Mg}^{2+}, 1,7 \mathrm{cmol}_{\mathrm{c}} / \mathrm{dm}^{3}$.

Foram realizadas aferições dos teores de umidade do solo na capacidade de campo de cada recipiente, com base no método gravimétrico. Cada recipiente teve sua superfície dividida igualmente em 11 linhas concêntricas, formando raios, enumerados de 1 a 11, correspondendo cada linha, aleatoriamente, a uma linhagem. Em cada linha foram consideradas seis covas para plantio. Foram semeadas três sementes por cova e, após sete dias, foram eliminadas as maiores e menores plantas, ficando, para cada linhagem, seis plantas por recipiente.

O delineamento experimental foi o de blocos casualizados no arranjo de parcelas subdivididas no tempo (subparcelas), com cinco repetições (Pimentel-Gomes, 1987). As avaliações da altura da planta e sintoma de murcha a intervalos de sete dias foram feitas pela manhã, entre $7 \mathrm{~h}$ e $10 \mathrm{~h}$.

O estudo compreendeu dois períodos de estresse hídrico. No primeiro período foram realizadas nove observações, compreendendo 63 dias, e no segundo, sete observações, com duração de 42 dias. Foram realizadas três irrigações, duas correspondentes ao início de cada período e outra ao final do segundo período de estresse. As irrigações foram realizadas simultaneamente em todos os recipientes, buscando simular uma chuva de 41,6 mm. Após cada irrigação não foi adicionada água até o início de um novo período. A segunda e terceira irrigações foram realizadas quando ocorria grande número de folhas com aspecto de palha seca (com a média das notas de sintomas de estresse hídrico aproximando-se de 85). Ao final de cada período, sete dias após a irrigação, registrou-se o porcentual de sobrevivência ao estresse, com a contagem das plantas.

As medições de altura de plantas foram realizadas com régua de escala em centímetros e medindo-se a extensão desde a base do caule até a curvatura da última folha completamente expandida. As atribuições das notas de sintomas de murcha foram baseadas no aspecto das folhas, utilizando-se a escala proposta por Reddy et al., citados por Lira et al. (1989): 0, ausência de murcha; 15, plântulas ligeiramente murchas no período da manhã; 50, plântulas muito murchas no período da manhã; 85 , grande número de folhas com aspecto de palha seca; 100 , plântulas com aspecto de palha seca.

Foram realizadas aferições de temperaturas máxima, mínima e atual, seguidas pelos registros de altura e atribuições das notas do raio 1 do recipiente 1 até o raio 11 do recipiente 5 , ininterruptamente, na ordem de recipiente. As médias das notas foram utilizadas como padrão para detectar o momento de irrigação, tornando-se parâmetro de grande importância na manutenção da capacidade de recuperação das linhagens mais resistentes. Ao final de cada período, a taxa de sobrevivência foi registrada no sétimo dia após a irrigação.

As médias dos parâmetros avaliados nos tratamentos foram comparadas pelo teste de Tukey a $5 \%$ de probabilidade.

\section{Resultados e Discussão}

A temperatura máxima variou de $34^{\circ} \mathrm{C}$ a $39^{\circ} \mathrm{C}$ e a mínima de $20^{\circ} \mathrm{C} \mathrm{a} 25^{\circ} \mathrm{C}$. Na superfície do solo, ao término de cada período, a temperatura apresentou oscilação entre $38^{\circ} \mathrm{C} \mathrm{e} 39^{\circ} \mathrm{C}$ no centro, e de $43^{\circ} \mathrm{C} \mathrm{a} 45^{\circ} \mathrm{C}$ nas bordas. Tais observações indicam aumento de até $12^{\circ} \mathrm{C}$ entre a temperatura ambiente na casa de vegetação e a temperatura na superfície do solo nas bordas do recipiente, provavelmente causado pelo material de confecção do recipiente reconhecidamente de alta retenção de calor.

Aos 8, 15 e 22 dias após o plantio, as linhagens não diferiram significativamente $(\mathrm{P}>0,05)$ quanto à altura média das plantas (Tabela 2). Aos 29 dias, a linhagem 33 apresentou altura maior $(\mathrm{P}<0,05)$ do que as linhagens 67, 92, 99 e 104, não revelando diferença $(\mathrm{P}>0,05)$ das demais. Entretanto, tanto a linhagem 33 como as demais só apresentaram aumento em altura até os 43 dias após o plantio; a partir desse dia os valores médios de altura das plantas foram reduzindo. Os efeitos do estresse hídrico tiveram início aos 29 dias após o plantio (Tabela 2), indicando a perda de turgescência das células, o que deve ter promovido o fechamento estomático, redução na assimilação de $\mathrm{CO}_{2}$ e inibição do processo fotossintético, tanto no que se refere ao transporte de elétrons como nos eventos bioquímicos e na atividade enzimática (Larcher, 1986; Taiz \& Zeiger, 1998).

No primeiro período, a linhagem 33 apresentou crescimento inicial mais acelerado, o que lhe confere, segundo Lira et al. (1989) e Taiz \& Zeiger (1998), características de suscetibilidade ao estresse hídrico. A linhagem 104 mostrou menor velocidade de crescimento nesta fase, conferindo-lhe características de resistência à seca, de acordo com Lira et al. (1989), os quais consideram que plantas com esse comportamento sofrem menos com o estresse hídrico. A redução de crescimento ocorreu, em média, após 
36 dias do início do experimento, evidenciada pela redução da altura média das plantas, sendo apontada como um dos principais sintomas do estresse hídrico de plantas (Ritchie, 1975). Tal comportamento também foi observado em Panicum maximum Jacq. (Dias Filho et al., 1989) e em Pennisetum purpureum Schum. (Barreto et al., 2001).

Houve diferenças significativas $(\mathrm{P}<0,05)$ entre as linhagens e as idades das plantas, com relação às notas atribuídas aos sintomas de murcha, no primeiro período (Tabela 3). Entretanto, a interação entre linhagens e idades não foi significativa $(\mathrm{P}>0,05)$. As plantas não apresentaram sintomas de estresse até os 22 dias, com notas zero para todas as linha- gens. Segundo Gomide et al. (1998), a magnitude dos efeitos de déficit hídrico depende muito da sua intensidade, duração e, principalmente, do momento de sua indução, com relação ao ciclo fenológico da cultura. A linhagem 33 apresentou as menores notas médias $(\mathrm{P}<0,05)$ dos 29 aos 43 dias, sendo inferior às linhagens 38 e 67 (29 dias), 40 (36 dias) e 38, 40 e 67 (43 dias). Aos 64 dias de idade, a média das notas das linhagens situava-se próximo de 85 , que corresponde a grande número de folhas com o aspecto de palha seca.

Os resultados do primeiro período indicam que o estresse hídrico do sorgo tornou-se severo após 36 dias do plantio, sendo o efeito constatado tanto

Tabela 2. Altura $(\mathrm{cm})$ média de plantas de linhagens de sorgo submetidas a estresse hídrico no primeiro período, que corresponde a observações realizadas a intervalos de sete dias ${ }^{(1)}$.

\begin{tabular}{cccccccccc}
\hline Linhagem & \multicolumn{10}{c}{ Dias após plantio } \\
\cline { 2 - 9 } & \multicolumn{10}{c}{15} & 22 & \multicolumn{10}{c}{29} & 36 & 43 & 50 & 57 & 64 \\
\hline 29 & $7,4 \mathrm{a}$ & $17,8 \mathrm{a}$ & $28,1 \mathrm{a}$ & $33,7 \mathrm{ab}$ & $42,8 \mathrm{ab}$ & $43,1 \mathrm{ab}$ & $39,8 \mathrm{a}$ & $37,6 \mathrm{a}$ & $31,5 \mathrm{ab}$ \\
33 & $8,5 \mathrm{a}$ & $18,5 \mathrm{a}$ & $29,9 \mathrm{a}$ & $43,1 \mathrm{a}$ & $48,0 \mathrm{a}$ & $48,7 \mathrm{a}$ & $41,0 \mathrm{a}$ & $38,5 \mathrm{a}$ & $34,3 \mathrm{a}$ \\
38 & $7,2 \mathrm{a}$ & $16,0 \mathrm{a}$ & $24,8 \mathrm{a}$ & $34,8 \mathrm{abc}$ & $38,7 \mathrm{abc}$ & $38,9 \mathrm{bc}$ & $33,1 \mathrm{ab}$ & $32,2 \mathrm{ab}$ & $27,9 \mathrm{ab}$ \\
40 & $5,9 \mathrm{a}$ & $17,0 \mathrm{a}$ & $25,1 \mathrm{a}$ & $34,9 \mathrm{abc}$ & $39,5 \mathrm{ab}$ & $36,3 \mathrm{bc}$ & $32,0 \mathrm{ab}$ & $27,9 \mathrm{~b}$ & $24,8 \mathrm{ab}$ \\
41 & $7,2 \mathrm{a}$ & $18,1 \mathrm{a}$ & $26,2 \mathrm{a}$ & $34,1 \mathrm{abc}$ & $38,7 \mathrm{abc}$ & $38,7 \mathrm{bc}$ & $32,0 \mathrm{ab}$ & $31,2 \mathrm{ab}$ & $27,2 \mathrm{ab}$ \\
63 & $7,2 \mathrm{a}$ & $18,2 \mathrm{a}$ & $26,9 \mathrm{a}$ & $38,7 \mathrm{ab}$ & $43,9 \mathrm{ab}$ & $44,3 \mathrm{ab}$ & $36,6 \mathrm{ab}$ & $35,5 \mathrm{ab}$ & $29,3 \mathrm{ab}$ \\
67 & $7,1 \mathrm{a}$ & $16,1 \mathrm{a}$ & $26,2 \mathrm{a}$ & $32.3 \mathrm{bc}$ & $37,5 \mathrm{bc}$ & $37,6 \mathrm{bc}$ & $32,5 \mathrm{ab}$ & $29,7 \mathrm{ab}$ & $25,0 \mathrm{ab}$ \\
84 & $6,6 \mathrm{a}$ & $16,5 \mathrm{a}$ & $25,5 \mathrm{a}$ & $35,0 \mathrm{abc}$ & $40,4 \mathrm{ab}$ & $40,8 \mathrm{abc}$ & $36,5 \mathrm{ab}$ & $34,8 \mathrm{ab}$ & $29,6 \mathrm{ab}$ \\
92 & $6,7 \mathrm{a}$ & $14,8 \mathrm{a}$ & $23,9 \mathrm{a}$ & $29,2 \mathrm{bc}$ & $36,1 \mathrm{bc}$ & $36,7 \mathrm{bc}$ & $33,1 \mathrm{ab}$ & $32,6 \mathrm{ab}$ & $25,9 \mathrm{ab}$ \\
99 & $6,9 \mathrm{a}$ & $16,1 \mathrm{a}$ & $25,6 \mathrm{a}$ & $30,7 \mathrm{bc}$ & $34,9 \mathrm{bc}$ & $35,3 \mathrm{bc}$ & $29,9 \mathrm{~b}$ & $28,1 \mathrm{~b}$ & $23,4 \mathrm{~b}$ \\
104 & $6,4 \mathrm{a}$ & $14,3 \mathrm{a}$ & $21,7 \mathrm{a}$ & $27,1 \mathrm{c}$ & $29,2 \mathrm{c}$ & $31,3 \mathrm{c}$ & $27,8 \mathrm{~b}$ & $26,6 \mathrm{~b}$ & $23,8 \mathrm{~b}$ \\
\hline
\end{tabular}

${ }^{(1)}$ Médias seguidas de mesma letra nas colunas não diferem entre si a 5\% de probabilidade, pelo teste de Tukey.

Tabela 3. Notas atribuídas aos sintomas de murcha em linhagens de sorgo submetidas a estresse hídrico no primeiro período, que corresponde a observações realizadas a intervalos de sete dias ${ }^{(1)}$.

\begin{tabular}{|c|c|c|c|c|c|c|c|c|c|}
\hline \multirow[t]{2}{*}{ Linhagem } & \multicolumn{9}{|c|}{ Dias após plantio } \\
\hline & 8 & 15 & 22 & 29 & 36 & 43 & 50 & 57 & 64 \\
\hline 29 & $0 \mathrm{a}$ & $0 \mathrm{a}$ & $0 \mathrm{a}$ & $18,7 \mathrm{ab}$ & $30,8 \mathrm{ab}$ & $54,0 \mathrm{ab}$ & $56,8 \mathrm{a}$ & $73,2 \mathrm{a}$ & $77,0 \mathrm{a}$ \\
\hline 33 & $0 \mathrm{a}$ & $0 \mathrm{a}$ & $0 \mathrm{a}$ & $8,0 \mathrm{~b}$ & $13,0 \mathrm{~b}$ & $39,3 b$ & $50,8 \mathrm{a}$ & $55,6 a$ & $79,6 a$ \\
\hline 38 & $0 \mathrm{a}$ & $0 \mathrm{a}$ & $0 \mathrm{a}$ & $23,8 \mathrm{a}$ & $35,1 \mathrm{ab}$ & $56,3 \mathrm{a}$ & $66,1 \mathrm{a}$ & $74,4 \mathrm{a}$ & $83,9 a$ \\
\hline 40 & 0a & $0 \mathrm{a}$ & $0 \mathrm{a}$ & $18,8 \mathrm{ab}$ & $36,7 \mathrm{a}$ & $59,1 \mathrm{a}$ & $70,3 a$ & $74,3 \mathrm{a}$ & $88,2 \mathrm{a}$ \\
\hline 41 & $0 \mathrm{a}$ & $0 \mathrm{a}$ & $0 \mathrm{a}$ & $17,3 \mathrm{ab}$ & $31,3 \mathrm{ab}$ & $55,6 a b$ & $68,8 \mathrm{a}$ & $68,8 \mathrm{a}$ & $80,8 \mathrm{a}$ \\
\hline 63 & $0 \mathrm{a}$ & $0 \mathrm{a}$ & $0 \mathrm{a}$ & $13,3 \mathrm{ab}$ & $22,6 \mathrm{ab}$ & $52,1 \mathrm{ab}$ & $54,1 \mathrm{a}$ & $66,3 a$ & $81,9 a$ \\
\hline 67 & $0 \mathrm{a}$ & $0 \mathrm{a}$ & $0 \mathrm{a}$ & $23,4 \mathrm{a}$ & $33,7 \mathrm{ab}$ & $60,5 a$ & $70,1 \mathrm{a}$ & $75,8 \mathrm{a}$ & $85,6 a$ \\
\hline 84 & $0 \mathrm{a}$ & $0 \mathrm{a}$ & $0 \mathrm{a}$ & $10,3 \mathrm{ab}$ & $18,5 \mathrm{ab}$ & $52,8 \mathrm{ab}$ & $63,2 \mathrm{a}$ & $70,7 \mathrm{a}$ & $77,0 \mathrm{a}$ \\
\hline 92 & $0 \mathrm{a}$ & $0 \mathrm{a}$ & $0 \mathrm{a}$ & $16,5 \mathrm{ab}$ & $32,4 \mathrm{ab}$ & $53,2 \mathrm{ab}$ & $60,5 a$ & $73,1 \mathrm{a}$ & $86,2 \mathrm{a}$ \\
\hline 99 & $0 \mathrm{a}$ & $0 \mathrm{a}$ & $0 \mathrm{a}$ & $13,9 \mathrm{ab}$ & $30,8 \mathrm{ab}$ & $50,9 \mathrm{ab}$ & $66,3 a$ & $75,0 \mathrm{a}$ & $84,1 \mathrm{a}$ \\
\hline 104 & $0 \mathrm{a}$ & $0 \mathrm{a}$ & $0 \mathrm{a}$ & $19,9 \mathrm{ab}$ & $34,1 \mathrm{ab}$ & $52,3 \mathrm{ab}$ & $69,8 \mathrm{a}$ & $73,5 \mathrm{a}$ & $87,0 \mathrm{a}$ \\
\hline
\end{tabular}

${ }^{(1)}$ Médias seguidas de mesma letra nas colunas não diferem entre si a 5\% de probabilidade, pelo teste de Tukey. 
pela redução de crescimento (Tabela 2) como pelos sintomas de seca (Tabela 3). Contudo, mesmo tendo ocorrido estresse de 28 dias, mais de $80 \%$ das plantas se recuperaram (Tabela 4) em cada tratamento, não havendo diferenças $(\mathrm{P}>0,05)$ entre as linhagens.

No segundo período (de 71 a 113 dias após o plantio), tanto a altura quanto a interação entre idade e linhagens não apresentaram diferenças significativas ( $\mathrm{P}>0,05)$ (Tabela 4). A análise de regressão indicou o ponto de máxima altura aos 87 dias; a partir daí as plantas reduziram o crescimento. Provavelmente, tal comportamento resultou de maior desenvolvimento das plantas no primeiro período, com um conseqüente maior requerimento hídrico.

Segundo a análise de regressão, aos 79 dias após o plantio, as plantas alcançaram a menor $(\mathrm{P}<0,05)$ nota média. Aos 113 dias de crescimento, idade em que as plantas foram irrigadas, as linhagens 33 e 40 apresentaram sintoma mais intenso do estresse hídrico, com notas médias de 97,4 e 95,4, respectivamente; as linhagens 92 e 84 apresentaram as menores notas médias, 78,7 e 82,8, respectivamente. A média geral atingiu a nota 89 , indicando necessidade de nova irrigação (Tabela 5).

$\mathrm{O}$ aparecimento de grande número de folhas secas, revelando necessidade de irrigação, ocorreu mais precocemente no primeiro período de avaliação, o que, provavelmente, está associado à necessidade de adaptação da planta a secas periódicas.
No primeiro período todas as plantas apresentaram elevada taxa de sobrevivência, variando de $80 \%$ a $100 \%$ (Tabela 6). A linhagem 29 apresentou $100 \%$ de sobrevivência aos 64 dias de deficiência hídrica. As linhagens 84 e 104 também apresentaram altas taxas de sobrevivência (97\%). A linhagem 92 apresentou a taxa de sobrevivência mais reduzida do período, com $80 \%$ das plantas superando o período de estresse. É possível que a ausência de diferenças nas taxas de sobrevivência seja decorrente de as linhagens de sorgo ocuparem o mesmo recipiente, já que as menos resistentes à seca utilizam mais rapidamente a água do que as mais resistentes (Lira et al., 1989). Assim, na competição com plantas suscetíveis, as resistentes consomem mais lentamente a água, todavia, retardam o crescimento, e não apresentam sintomas visuais de murcha. Contudo, essas plantas provavelmente têm seu aparato fisiológico comprometido, o que pode ser comprovado pela alta taxa de mortalidade da linhagem 92.

No segundo período, a taxa de sobrevivência variou de $27 \%$ a $50 \%$, tendo a linhagem 84 a mais alta taxa, seguida pelas linhagens 29 e 99, com taxa de $47 \%$, mantendo todas citadas o comportamento do período anterior. Segundo Ortolani \& Camargo (1987), a deficiência hídrica provoca alterações cuja irreversibilidade no comportamento vegetal depende da duração e severidade do estresse, do estádio de desenvolvimento da planta, da história prévia do

Tabela 4. Altura $(\mathrm{cm})$ média de planta em linhagens de sorgo submetidas a estresse hídrico no segundo período, que corresponde a observações realizadas a intervalos de sete dias ${ }^{(1)}$.

\begin{tabular}{rccccccc}
\hline Linhagem & \multicolumn{7}{c}{ Dias após plantio } \\
\cline { 2 - 7 } & 71 & 78 & 85 & 92 & 99 & 106 & 113 \\
\hline 29 & $34,4 \mathrm{a}$ & $39,8 \mathrm{a}$ & $44,4 \mathrm{a}$ & $43,3 \mathrm{a}$ & $40,1 \mathrm{a}$ & $34,4 \mathrm{a}$ & $27,9 \mathrm{a}$ \\
33 & $34,7 \mathrm{a}$ & $38,1 \mathrm{a}$ & $38,0 \mathrm{a}$ & $33,7 \mathrm{a}$ & $27,9 \mathrm{a}$ & $25,5 \mathrm{a}$ & $22,2 \mathrm{a}$ \\
38 & $29,5 \mathrm{a}$ & $30,4 \mathrm{a}$ & $33,8 \mathrm{a}$ & $38,0 \mathrm{a}$ & $36,8 \mathrm{a}$ & $34,6 \mathrm{a}$ & $31,4 \mathrm{a}$ \\
40 & $23,7 \mathrm{a}$ & $24,2 \mathrm{a}$ & $27,9 \mathrm{a}$ & $23,3 \mathrm{a}$ & $20,3 \mathrm{a}$ & $22,4 \mathrm{a}$ & $17,5 \mathrm{a}$ \\
41 & $32,9 \mathrm{a}$ & $34,7 \mathrm{a}$ & $34,0 \mathrm{a}$ & $33,6 \mathrm{a}$ & $29,3 \mathrm{a}$ & $28,1 \mathrm{a}$ & $24,0 \mathrm{a}$ \\
63 & $35,2 \mathrm{a}$ & $37,9 \mathrm{a}$ & $40,4 \mathrm{a}$ & $34,5 \mathrm{a}$ & $31,6 \mathrm{a}$ & $28,5 \mathrm{a}$ & $24,2 \mathrm{a}$ \\
67 & $28,6 \mathrm{a}$ & $33,6 \mathrm{a}$ & $39,9 \mathrm{a}$ & $36,6 \mathrm{a}$ & $33,5 \mathrm{a}$ & $34,7 \mathrm{a}$ & $30,0 \mathrm{a}$ \\
84 & $35,8 \mathrm{a}$ & $35,7 \mathrm{a}$ & $37,9 \mathrm{a}$ & $37,3 \mathrm{a}$ & $36,3 \mathrm{a}$ & $29,3 \mathrm{a}$ & $24,5 \mathrm{a}$ \\
92 & $30,7 \mathrm{a}$ & $33,6 \mathrm{a}$ & $41,7 \mathrm{a}$ & $41,7 \mathrm{a}$ & $38,2 \mathrm{a}$ & $36,6 \mathrm{a}$ & $30,1 \mathrm{a}$ \\
99 & $27,6 \mathrm{a}$ & $31,7 \mathrm{a}$ & $35,1 \mathrm{a}$ & $34,6 \mathrm{a}$ & $32,7 \mathrm{a}$ & $27,7 \mathrm{a}$ & $23,4 \mathrm{a}$ \\
104 & $27,7 \mathrm{a}$ & $28,6 \mathrm{a}$ & $28,6 \mathrm{a}$ & $30,3 \mathrm{a}$ & $28,0 \mathrm{a}$ & $27,1 \mathrm{a}$ & $21,3 \mathrm{a}$ \\
\hline
\end{tabular}

${ }^{(1)}$ Médias seguidas de mesma letra nas colunas não diferem entre si a $5 \%$ de probabilidade, pelo teste de Tukey. 
Tabela 5. Notas atribuídas aos sintomas de murcha em linhagens de sorgo submetidas a estresse hídrico no segundo período, que corresponde a observações realizadas a intervalos de sete dias ${ }^{(1)}$.

\begin{tabular}{cccccccc}
\hline \multirow{2}{*}{ inhagem } & \multicolumn{7}{c}{ Dias após plantio } \\
\cline { 2 - 7 } & 71 & 78 & 85 & 92 & 99 & 106 & 113 \\
\hline 29 & $22,3 \mathrm{a}$ & $16,5 \mathrm{a}$ & $24,7 \mathrm{a}$ & $37,8 \mathrm{a}$ & $54,7 \mathrm{a}$ & $64,0 \mathrm{a}$ & $90,7 \mathrm{a}$ \\
33 & $20,8 \mathrm{a}$ & $13,8 \mathrm{a}$ & $21,0 \mathrm{a}$ & $36,0 \mathrm{a}$ & $60,7 \mathrm{a}$ & $80,1 \mathrm{a}$ & $97,4 \mathrm{a}$ \\
38 & $41,0 \mathrm{a}$ & $41,2 \mathrm{a}$ & $28,1 \mathrm{a}$ & $32,1 \mathrm{a}$ & $47,9 \mathrm{a}$ & $66,7 \mathrm{a}$ & $90,7 \mathrm{a}$ \\
40 & $41,2 \mathrm{a}$ & $38,5 \mathrm{a}$ & $27,5 \mathrm{a}$ & $42,4 \mathrm{a}$ & $58,5 \mathrm{a}$ & $70,3 \mathrm{a}$ & $95,5 \mathrm{a}$ \\
41 & $25,6 \mathrm{a}$ & $14,4 \mathrm{a}$ & $23,9 \mathrm{a}$ & $34,7 \mathrm{a}$ & $49,8 \mathrm{a}$ & $70,3 \mathrm{a}$ & $91,3 \mathrm{a}$ \\
63 & $29,3 \mathrm{a}$ & $18,1 \mathrm{a}$ & $13,8 \mathrm{a}$ & $24,6 \mathrm{a}$ & $55,1 \mathrm{a}$ & $69,8 \mathrm{a}$ & $90,4 \mathrm{a}$ \\
67 & $44,4 \mathrm{a}$ & $35,8 \mathrm{a}$ & $22,7 \mathrm{a}$ & $29,6 \mathrm{a}$ & $51,0 \mathrm{a}$ & $72,4 \mathrm{a}$ & $88,8 \mathrm{a}$ \\
84 & $20,7 \mathrm{a}$ & $18,8 \mathrm{a}$ & $30,2 \mathrm{a}$ & $34,6 \mathrm{a}$ & $44,4 \mathrm{a}$ & $66,2 \mathrm{a}$ & $82,8 \mathrm{a}$ \\
92 & $33,8 \mathrm{a}$ & $21,3 \mathrm{a}$ & $10,3 \mathrm{a}$ & $23,7 \mathrm{a}$ & $49,4 \mathrm{a}$ & $54,2 \mathrm{a}$ & $78,7 \mathrm{a}$ \\
99 & $31,0 \mathrm{a}$ & $13,8 \mathrm{a}$ & $17,5 \mathrm{a}$ & $24,3 \mathrm{a}$ & $48,9 \mathrm{a}$ & $69,9 \mathrm{a}$ & $89,3 \mathrm{a}$ \\
104 & $24,2 \mathrm{a}$ & $16,0 \mathrm{a}$ & $26,5 \mathrm{a}$ & $36,6 \mathrm{a}$ & $59,9 \mathrm{a}$ & $73,7 \mathrm{a}$ & $88,6 \mathrm{a}$ \\
\hline
\end{tabular}

${ }^{(1)}$ Médias seguidas de mesma letra nas colunas não diferem entre si a 5\% de probabilidade, pelo teste de Tukey.

Tabela 6. Número (NP) e taxa de sobrevivência (TS) de plantas em linhagens de sorgo submetidas a dois períodos de estresse hídrico, avaliadas sete dias após irrigação( ${ }^{(1)}$.

\begin{tabular}{cccrrc}
\hline Linhagem & \multicolumn{2}{c}{ Período 1 } & & \multicolumn{2}{c}{ Período 2 } \\
\cline { 2 - 3 } \cline { 5 - 6 } & NP & TS (\%) & & NP & TS (\%) \\
\hline 29 & 30 & 100 & & 14 & 47 \\
33 & 26 & 87 & & 11 & 37 \\
38 & 26 & 87 & & 9 & 30 \\
40 & 28 & 93 & & 8 & 27 \\
41 & 27 & 90 & & 13 & 43 \\
63 & 25 & 83 & & 13 & 43 \\
67 & 26 & 87 & & 8 & 27 \\
84 & 29 & 97 & & 15 & 50 \\
92 & 24 & 80 & & 12 & 40 \\
99 & 28 & 93 & & 14 & 47 \\
104 & 29 & 97 & & 13 & 43 \\
\hline
\end{tabular}

(1)Período 1: nove observações realizadas a intervalos de sete dias; período 2: sete observações realizadas a intervalos de sete dias.

estresse e do genótipo. A menor taxa de sobrevivência nesse período correspondeu às linhagens 40 e 67 , ambas com $27 \%$ de plantas sobreviventes (Tabela 6).

\section{Conclusões}

1. As linhagens apresentam alta capacidade de resistência ao estresse hídrico na fase inicial de crescimento.

2. A velocidade de crescimento não diferencia satisfatoriamente linhagens de comportamentos distintos, quanto à resistência à seca.
3. As linhagens $84,29,99,41,63$ e 104 são as mais resistentes ao estresse hídrico e as linhagens 33, 38, 40,67 e 92 as menos resistentes.

\section{Referências}

BARRETO, G. P.; LIRA, M. de A.; SANTOS, M. V. F. dos S.; DUBEUX JÚNIOR, J. C. B. Avaliação de clones de capim elefante (Pennisetum purpureum Schum.) e de um híbrido com milheto (Pennisetum glaucum (L.) R. Br.) submetidos a estresse hídrico - 1: parâmetros morfológicos. Revista Brasileira de Zootecnia, Viçosa, MG, v. 30, n. 1, p. 1-6, 2001.

DIAS FILHO, M. B.; CORSI, M.; CUSATO, S. Respostas morfológicas de Panicum maximum Jacq. cv. Tobiatã ao estresse hídrico. Pesquisa Agropecuária Brasileira, Brasília, v. 24, n. 7, p. 893-898, jul. 1989.

DONATELLI, M.; HAMMER, G. L.; VANDERLIP, R. L. Genotype and water limitation effects on phenology, growth and transpiration efficiency in grain sorghum. Crop Science, Madison, v. 32, p. 781-786, 1992.

GOMIDE, R. L.; MAGALHÃES, P. C.; WAQUIL, J. M.; FERREIRA, W. P. Avaliação do estresse hídrico em cultivares de milho e sorgo por meio de um gradiente contínuo de irrigação. In: CONGRESSO BRASILEIRO DE MILHO E SORGO, 22., 1998, Recife. Anais... Recife: Empresa Pernambucana de Pesquisa Agropecuária/EmbrapaCNPMS, 1998. 4 p. CD-ROM. 
LARCHER, W. Ecofisiologia vegetal. São Paulo: EPU, 1986. $319 \mathrm{p}$.

LIRA, M. de A.; BRANDÃO, A. R. M.; TABOSA, J. N.; BRITO, G. Q. Estudos preliminares de resistência à seca em genótipos de sorgo forrageiro (Sorghum bicolor (L.) Moench). Revista da Sociedade Brasileira de Zootecnia, Viçosa, MG, v. 18, n. 1, p. 1-12, 1989.

MASOJIDEK, J.; TRIVEDI, S.; HALSHAW, L.; ALEXIOU, A.; HALL, D. O. The synergetic effect of drought and light stress in sorghum and pearl millet. Plant Physiology, Bethesda, v. 96, p. 198-207, 1991.

OLIVEIRA, J. S.; FERREIRA, R. P.; CRUZ, C. D.; PEREIRA, A. V.; BOTREL, M. A.; VON PINHO, R. G.; RODRIGUES, J. A. S.; LOPES, F. C. F.; MIRANDA, J. E. C. Adaptabilidade e estabilidade em cultivares de sorgo. Revista Brasileira de Zootecnia, Viçosa, MG, v. 31 , n. 2 , p. $883-889,2002$. Suplemento.
ORTOLANI, A. A.; CAMARGO, M. B. P. Influência dos fatores climáticos na produção. In: CASTRO, P. R. C.; FERREIRA, S. O.; YAMADA, T. (Ed.). Ecofisiologia da produção agrícola. Piracicaba: Potafos, 1987. p. 71-81.

PIMENTEL-GOMES, F. Curso de estatística experimental. Piracicaba: Nobel, 1987. 467 p.

REIS, O. V. Seleção de linhagens de sorgo forrageiro (Sorghum bicolor (L.) Moench) tolerantes ao estresse hídrico em fase de plântula. 1992. 150 f. Dissertação (Mestrado em Botânica) - Universidade Federal Rural de Pernambuco, Recife, 1992.

RITCHIE, J. T. Atmospheric and soil water influences on the plant water balance. In: STONE, J. F. (Ed.). Plant modification for more efficient water use. Amsterdam: Elsevier Scientific, 1975. p. 183-198.

TAIZ, L.; ZEIGER, E. Plant physiology. $2^{\text {nd }}$ ed. Sunderland: Sinauer, 1998. 792 p. 